\title{
OPEN A multichannel color filter with the functions of optical sensor and switch
}

\author{
Yuan-Fong Chou Chau ${ }^{1 凶}$, Chung-Ting Chou Chao², Hung Ji Huang ${ }^{3}$, Sy-Hann Chen ${ }^{4}$, \\ Tsung Sheng $\mathrm{KaO}^{5 \bowtie}$ \& Hai-Pang Chiang ${ }^{2 \bowtie}$
}

This paper reports a multichannel color filter with the functions of optical sensor and switch. The proposed structure comprises a metal-insulator-metal (MIM) bus waveguide side-couples to six circular cavities with different sizes for filtering ultra-violet and visible lights into individual colors in the wavelength range of $350-700 \mathrm{~nm}$. We used the finite element method to analyze the electromagnetic field distributions and transmittance properties by varying the structural parameters in detail. The designed plasmonic filter takes advantage of filtering out different colors since the lightmatter resonance and interference between the surface plasmon polaritons (SPPs) modes within the six cavities. Results show that the designed structure can preferentially select the desired colors and confine the SPPS modes in one of the cavities. This designed structure can filter eleven color channels with a small full width at half maximum (FWHM) $2 \mathrm{~nm}$. Furthermore, the maximum values of sensitivity, figure of merit, quality factor, dipping strength, and extinction ratio can achieve of $700 \mathrm{~nm} /$ RIU, 350 1/RIU, 349.0, 65.04\%, and $174.50 \mathrm{~dB}$, respectively, revealing the excellent functions of sensor performance and optical switch, and offering a chance for designing a beneficial nanophotonic device.

Surface plasmon polaritons (SPPs) are the amplification of electromagnetic (EM) waves since the interaction of incident photons with conduction electrons of metal nanoparticles (MNPs) on the surface of the metal-dielectric boundary ${ }^{1-7}$. The light-matter interaction depends on the dimension, volume, aspect of the MNPs, and the origin and constitution of the dispersion material ${ }^{8-10}$. SPPs waves solve the problem of the light diffraction limit and could confine the light within nanometer scale; consequently, they have broad-ranging applications of SPPs wave in integrated optical circuits (IOCs) and optical devices ${ }^{11-15}$, such as absorbers ${ }^{16,17}$, modulators ${ }^{18,19}$, splitters ${ }^{20}$, amplifiers $^{21,22}$, switches ${ }^{23,24}$, filters ${ }^{25,26}$, sensors ${ }^{27-31}$ and so forth. Among them are metal-insulator-metal (MIM) waveguide-cavity-based devices with long propagation distances $(1-40 \mu \mathrm{m})$, strong EM wave confinement, inexpensive production, low loss, and ease of fabrication and integration have attracted considerable interest and consideration ${ }^{32-35}$.

By comprehending the nature outlines of the light-matter coupling of changed MNP geometries and the corresponding material effect, new approaches have been exploited for cautious spectroscopic inspecting. Each variation can be manipulated by various spectroscopic techniques, which result in imaging events and sensing applications. From successful SPP mode monitoring through spectroscopy, new nanophotonic devices, including color sensors and devise sensor elements, have been investigated and developed ${ }^{36}$. Color filters can cope with precise wavelengths of concern or filter ultra-violet and visible light into separate colors or wavelengths. Nowadays, color filters are essential in many imaging devices, such as display units of computerized systems, digital projectors, digital photography, organic light-emitting diodes, etc. ${ }^{37,38}$. Researchers are consecutively looking for a scheme to create low-price, compact, and transmission effectual color filters to improve the traditional pigment-based printing. Recent advancements in manufacturing MNP-based structures have exhibited a method to surmount the diffraction and reach nano-size resolution ${ }^{39}$. One such technique can utilize plasmonic cavities merging SPPs, which can couple incident light into EM modes transferring onto the MIM surface ${ }^{40,41}$.

\footnotetext{
${ }^{1}$ Centre for Advanced Material and Energy Sciences, Universiti Brunei Darussalam, Tungku Link, Gadong BE1410, Brunei Darussalam. ${ }^{2}$ Department of Optoelectronics and Materials Technology, National Taiwan Ocean University, Keelung 20224, Taiwan, ROC. ${ }^{3}$ Taiwan Instrument Research Institute, National Applied Research Laboratories, Hsinchu 300, Taiwan, ROC. "Department of Electrophysics, National Chiayi University, 600, Chiayi, Taiwan, ROC. ${ }^{5}$ Department of Photonics \& Institute of Electro-Optical Engineering, College of Electrical and Computer Engineering, National Yang Ming Chiao Tung University, Hsinchu 300, Taiwan, ROC. ${ }^{\circledR}$ email: chou.fong@ ubd.edu.bn; tskao@nctu.edu.tw; hpchiang@mail.ntou.edu.tw
} 


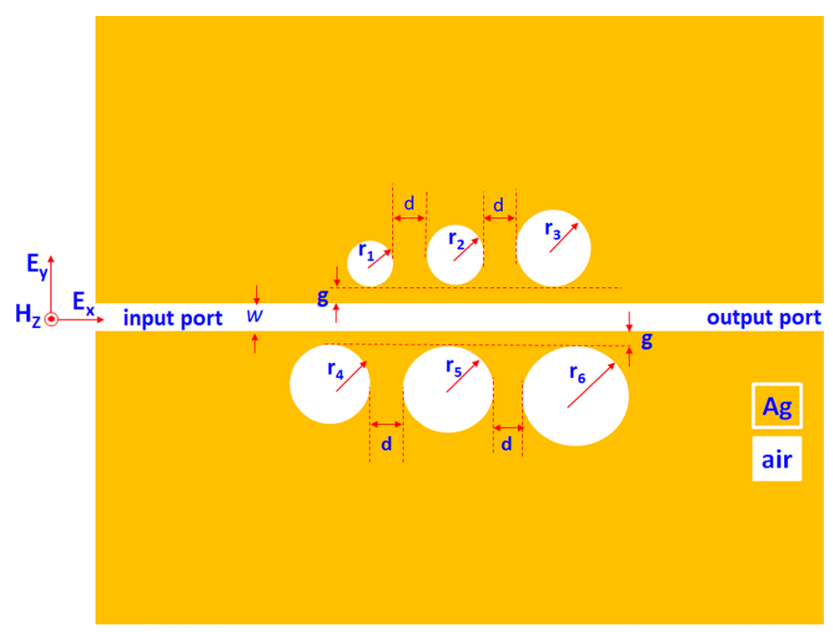

Figure 1. Top view of the proposed color filter, consisting of a MIM bus waveguide side-coupled with six circular cavities.

Recently, several research groups exploited the plasmonic color filters based on SPPs through different MIMcavity-based configurations. In Ref. ${ }^{37}$, Zhang and coauthors proposed a multiband four-channel color filter employing an elliptical-shaped cavity and obtained the maximum sensitivity of $608 \mathrm{~nm} / \mathrm{RIU}$ and figure of merit (FOM) of 105.02. Diest et al. ${ }^{38}$ experimentally and numerically demonstrated that arranged two slits into the waveguide and distinguished three primary colors, i.e., red, green, and blue. Combining six $\mu$-ring cavities, Butt et al. ${ }^{42}$ partitioned the visible light into six colors and claimed that the highest sensitivity and figure of merit in mode 1 (i.e., at the largest resonance wavelength) could reach $700 \mathrm{~nm} / \mathrm{RIU}$ and $191.6 \mathrm{1} / \mathrm{RIU}$, respectively. However, the transmittance spectral of these articles show a higher full width at half maximum (FWHM) and a smaller dipping strength, which influence the resolution in color filtering and the performance of refractive index (RI) detecting.

This work proposed a novel and simple frame of the plasmonic color filter based on MIM-cavity configuration employing one bus waveguide side-coupled to six circular cavities. We used the two-dimensional (2-D) finite element method to analyze the EM field distributions and transmittance resonance modes by varying the structural parameters in detail. The introduction of six circular cavities near the MIM bus waveguide acting as resonators can remarkably improve the sensing performance because of their excellent optical features in the EM field confinement and low Ohmic loss. In addition, the resonance modes or channels found in the cavities have a significant impact on the coupling efficiency between the bus waveguide and resonance cavities, which is less discussed before and is needed to investigate further. The designed plasmonic filter can separate eleven color channels with a small full width at half maximum (FWHM) $\sim 2 \mathrm{~nm}$ in the ultraviolet and visible ranges, which cannot attain in other reported articles.

Furthermore, six air cavities can preferentially select the desired colors (or wavelengths) and confine the SPPS modes in one of the cavities. In addition, the designed optical filter can function as an optical sensor and an alloptical switch, which possess the merit of excellent sensing performance and a high extinction ratio. Compared to the reported color filters, the proposed structure is straightforward and is rarely investigated before. The achieved plasmonic sensor is ideal for color detection design and can apply in nanophotonic devices, such as RI sensors and optical switches, and the range of the detected RI is available for testing RI materials.

\section{Structure model, simulation method, and fundamental}

Figure 1 shows the top view of the investigated color filter, consisting of a MIM bus waveguide side-coupled with six circular cavities (with the radius of $r_{1}, r_{2}, r_{3}, r_{4}, r_{5}$, and $r_{6}$ ) at both sides of the bus waveguide. We indicated the structural parameters in Fig. 1, i.e., the width of the bus waveguide is $w$, the gap between the bus waveguide and the six circular ring-shaped cavities is $g$, the space between each circular cavity is $d$, respectively. In Fig. 1, the golden- and white-colored regions signify the silver and insulator medium (air with the RI of $n=1.00$ ), respectively. We used a FEM-based commercial software, COMSOL Multiphysics, to calculate the transmittance spectrum and EM field distribution. The perfect matched layer boundary condition to form a border to avoid EM wave's reflections and extra-fine triangular meshing for the discretization of the simulation domains. The proposed color filter in the $z$-direction is infinite compared to the $\mathrm{x}$ - and $\mathrm{y}$-axes. Thus, the simulation model is a 2-D one since a three-dimensional (3-D) model can obtain similar results in simulations $s^{43}$ and experiments ${ }^{44}$. Besides, the 2-D simulations can save the computer resources ${ }^{45}$ without sacrificing the calculation precision. A TM-polarized EM wave with incident wavelength ranging in 350-700 nm coupled with the fundamental SPP mode ${ }^{46-48}$ into the bus waveguide's input port ${ }^{49,50}$.

Among all metals, silver $(\mathrm{Ag})$ and gold $(\mathrm{Au})$ have been the top choices for researchers due to numerous advantages $^{51}$, providing satisfactory plasmonic response at optical frequencies. We choose silver as the plasmonic material because it can generate an EM wave response within the ultra-violet and visible range and exhibits the most negligible Drude damping, the small imaginary part of the relative permittivity, lower cost-consuming, and 


\begin{tabular}{|l|l|l|l|l|l|l|l|l|}
\hline $\boldsymbol{r}_{\boldsymbol{1}}(\mathbf{n m})$ & $\boldsymbol{r}_{2}(\mathbf{n m})$ & $\boldsymbol{r}_{3}(\mathbf{n m})$ & $\boldsymbol{r}_{4}(\mathbf{n m})$ & $\boldsymbol{r}_{5}(\mathbf{n m})$ & $\boldsymbol{r}_{\mathbf{6}}(\mathbf{n m})$ & $\boldsymbol{w}(\mathbf{n m})$ & $\boldsymbol{g}(\mathbf{n m})$ & $\boldsymbol{d}(\mathbf{n m})$ \\
\hline 80 & 100 & 120 & 132 & 140 & 160 & 100 & 40 & 100 \\
\hline
\end{tabular}

Table 1. Default geometrical parameters of the proposed color filter.

less power consumption. In addition, the other stable metals (e.g., Au, copper, or Pt) can also use in simulations and fabrication. The relative permittivity $\left(\varepsilon_{\mathrm{m}}\right)$ of $\mathrm{Ag}$ can characterize by the Drude model ${ }^{52}$

$$
\varepsilon_{\mathrm{m}}(\omega)=\varepsilon_{\infty}-\frac{\omega_{\mathrm{p}}^{2}}{\omega^{2}+i \omega \gamma}
$$

where $\omega$ is the frequency, $\omega_{\mathrm{p}}=9.10 \mathrm{eV}, \varepsilon_{\infty}=3.7$ and $\gamma=18 \mathrm{meV}$ are bulk plasma frequency, infinite dielectric constant, and electron collision frequency, respectively. The transmittance $(\mathrm{T})$ can calculate as $\mathrm{T}=\mathrm{P}_{\text {out }}$ (output power $) / \mathrm{P}_{\text {in }}$ (input power). The resonance modes in the individual cavity will occur when the SPPs from the bus waveguide coupled to the circular pits match the resonance condition. If $\Delta \varphi=2 \pi j$ ( $j$ is an integer), the resonance wavelength $\left(\lambda_{\text {res }}\right)$ can be expressed by ${ }^{53,54}$

$$
\lambda_{\text {res }}=\frac{2 L_{e f f} \operatorname{Re}\left(n_{\mathrm{eff}}\right)}{j-\frac{\varphi}{2 \pi}}(j=1,2,3 \ldots)
$$

here, $j$ denotes the order of the standing wave resonance, $L_{\text {eff }}$ represents the cavity's effective length, $\varphi$ stands for the phase shift, and $\operatorname{Re}\left(n_{\text {eff }}\right)$ is the effective RI's real part. $n_{\text {eff }}$ can describe as:

$$
\operatorname{Re}\left(n_{\mathrm{eff}}\right)=\left(\varepsilon_{\text {metal }}+\left(\frac{k}{k_{0}}\right)^{2}\right)^{\frac{1}{2}}
$$

$\mathrm{k}=2 \pi / \lambda$ and $k_{0}$ are the wave vector in material and free space, respectively.

Sensitivity (S) and figure of merit (FOM) are two essential factors for sensing design. We use $S=\Delta \lambda / \Delta n(\mathrm{~nm} /$ RIU, RIU is a RI unit) and FOM $=S$ /FWHM to calculate the $S$ and FOM, where $\Delta \lambda$ is the $\lambda_{\text {res }}$ shift of transmittance, and $\Delta n$ is the difference in the RI corresponding to $\lambda_{\text {res }}$. Full width at half-maximum (FWHM) can define as the bandwidth value connected to the left and right of the half-high position of the transmittance spectrum. Besides, we can obtain quality (Q) factor using $\lambda_{\text {res }} / F W H M$.

The proposed color filter can manufacture using stripping and electric beam lithography (EBL) processes ${ }^{55}$, which can imprint custom pictures with sub-10 nm resolution ${ }^{51}$. The fabrication of the proposed color filter is a straightforward process. First, a thin layer of Ag can deposit on a silica substrate followed by EBL patterning of the design. Then, one can use wet etching (e.g., diluted nitric acid) to eliminate the undesired Ag through lift-off. However, the purpose of this article does not to concentrate on the fabricating process. As a substitute, some potential papers that focus on this theme are advised ${ }^{56-58}$.

\section{Design of a multichannel plasmonic color filter}

For simplicity, we do not discuss optimizing geometrical parameters on the proposed color filter but directly provide the default values, as shown in Table 1. Figure 2 depicts the transmittance spectrum of the SPPs mode for the proposed structure. Here, we define the dipping strength $(\Delta \mathrm{D})$ as the difference between the maximum and minimum transmittance (i.e., $\left.\Delta \mathrm{D}=\left(\mathrm{T}_{\max }-\mathrm{T}_{\min }\right) \times 100 \%\right)^{59}$.

In Eq. (2), $\lambda_{\text {res }}$ is proportional to $L_{\text {eff, }}$, while the effective RI $\left(n_{\text {eff }}\right)$ and mode integer $j$ are the same. One can easily detect color if the $\lambda_{\text {res }}$ is recognized. The ultraviolet and visible light impinges into the input end of the bus waveguide side-coupled to the six circular cavities when the resonance condition in the circular cavities is satisfied. As shown in Fig. 2, eleven sharp transmittance dips corresponding to the different resonance modes (i.e., mode 1 to mode 11 or channel 1 to channel 11) distribute in the range of visible and ultraviolet, which accords with red $\left(\lambda_{\text {res }}=666.5 \mathrm{~nm}\right)$, orange $\left(\lambda_{\text {res }}=601.5 \mathrm{~nm}\right)$, yellow $\left(\lambda_{\text {res }}=575 \mathrm{~nm}\right)$, green $\left(\lambda_{\text {res }}=537.5 \mathrm{~nm}\right)$, blue $\left(\lambda_{\text {res }}=476.5 \mathrm{~nm}\right)$, violet $\left(\lambda_{\text {res }}=439,420,406,393 \mathrm{~nm}\right)$, and ultraviolet (377 and $\left.363 \mathrm{~nm}\right)$ colors, respectively. Therefore, one can establish the detection of those specific colors ranging in ultraviolet and visible. These SPP modes are due to the cavity plasmon resonance (CPR) and surface plasmon resonance (SPR) arising from the coupling effect between the six circular cavities and the bus waveguide ${ }^{60}$. The interference of SPR and CPR cause the multichannel SPPs modes between bus waveguides and circular cavities ${ }^{8,61,62}$, leading to eleven available SPPs modes in the wavelength of $350-700 \mathrm{~nm}$. We found that CPR plays a pivotal role in offering more plasmon resonance in the proposed color filter system. The obtained transmittance dips possess a more profound dipping strength $(\Delta \mathrm{D})$ ranging in $53.28-66.71 \%$ and a narrower FWHM of $\sim 2 \mathrm{~nm}$ compared to the reported articles (e.g. ${ }^{37,42}$ ), both beneficial to the resolution of the color filter and the performance of RI sensor. It is worth noting that four and two channels ranging in violet and ultraviolet lights appeared in the proposed color filter, which the previous articles cannot attain (e.g. ${ }^{37,38,42}$ ). Violet and ultraviolet rays are prioritized in this scenario as recent works studied the application of the violet light in various cases such as surgical management ${ }^{63}$, dental bleaching ${ }^{64}$, fungal study ${ }^{65}$, and forensic science ${ }^{66}$. This remarkable feature of the designed filter has led to potential applications in the medical field.

When the incident EM wave satisfies the resonance condition in the optical filter system, the SPPs' energy delivered from the bus waveguide to the six cavities through near field coupling. It allows constructing a stable 


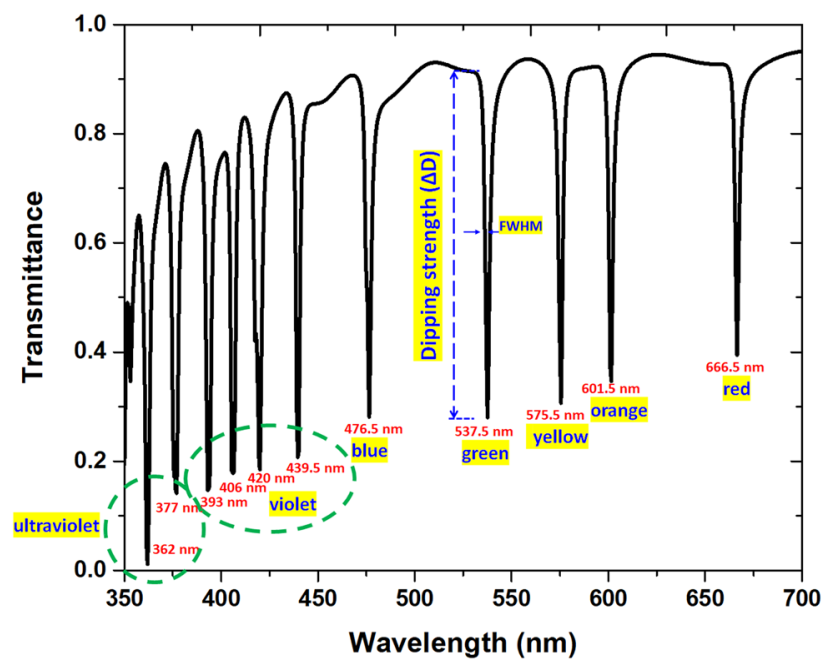

Figure 2. Transmittance spectrum of the proposed color filter structure ranging in $350-700 \mathrm{~nm}$.
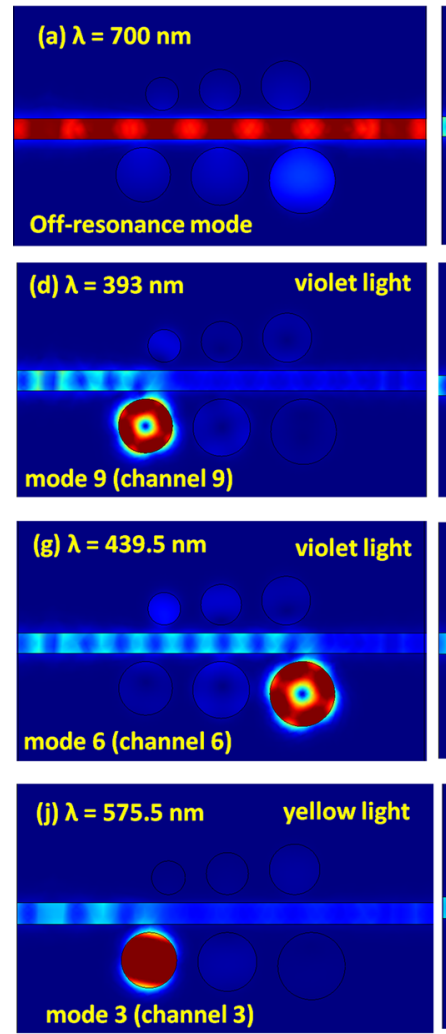
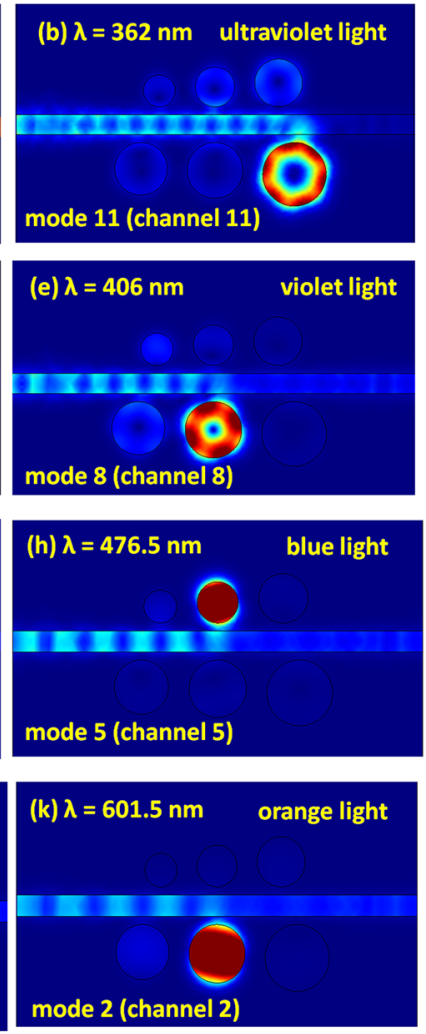

0
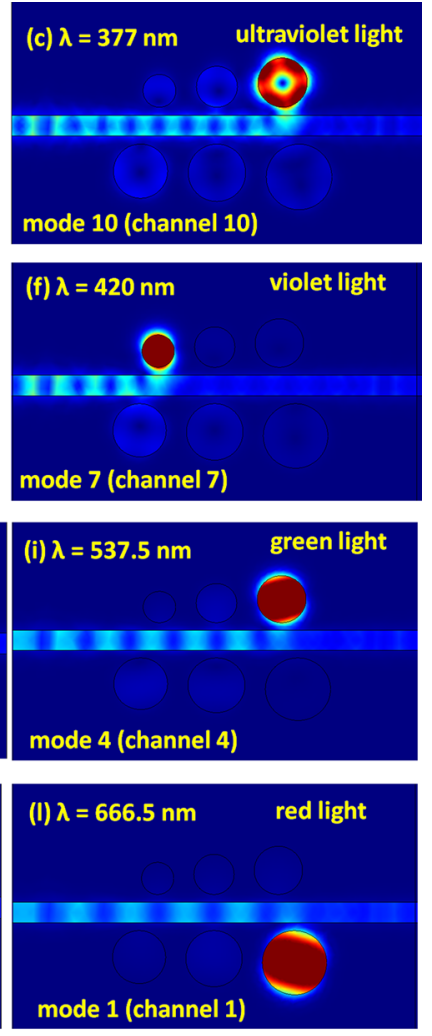

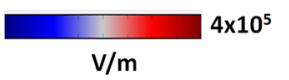

Figure 3. Truncate views of electric field intensity $(|E|)$ distributions at one of the off-resonance wavelengths $\left(\lambda_{\text {res }}=700 \mathrm{~nm}\right)$ and eleven resonance wavelengths $\left(\lambda_{\text {res }}=362,377,393,406,420,439.5,476.5,537.5,575.5,601.5\right.$ and $666.5 \mathrm{~nm}$ ) from channel 1 to channel 11 (i.e., mode 1 -mode 11), respectively.

standing wave mode in the six cavities, which can productively engineer the profile of the transmittance spectrum. To comprehend the physical mechanism of resonance modes that occurred in the investigated filter system, Fig. 3a-l illustrate the electric field intensity $(|\mathrm{E}|)$ distributions at one of off-resonance wavelengths $\left(\lambda_{\text {res }}=700 \mathrm{~nm}\right)$ and eleven resonance wavelengths $\left(\lambda_{\text {res }}=362,377,393,406,420,439.5,476.5,537.5,575.5,601.5\right.$ and $\left.666.5 \mathrm{~nm}\right)$ from channel 1 to channel 11 (i.e., mode 1 to mode 11), respectively. The wavelengths of EM waves with different phases associate with the energy and establish the apparent color. When $\lambda_{\text {res }}$ at off-resonance mode (Fig. 3a), the 

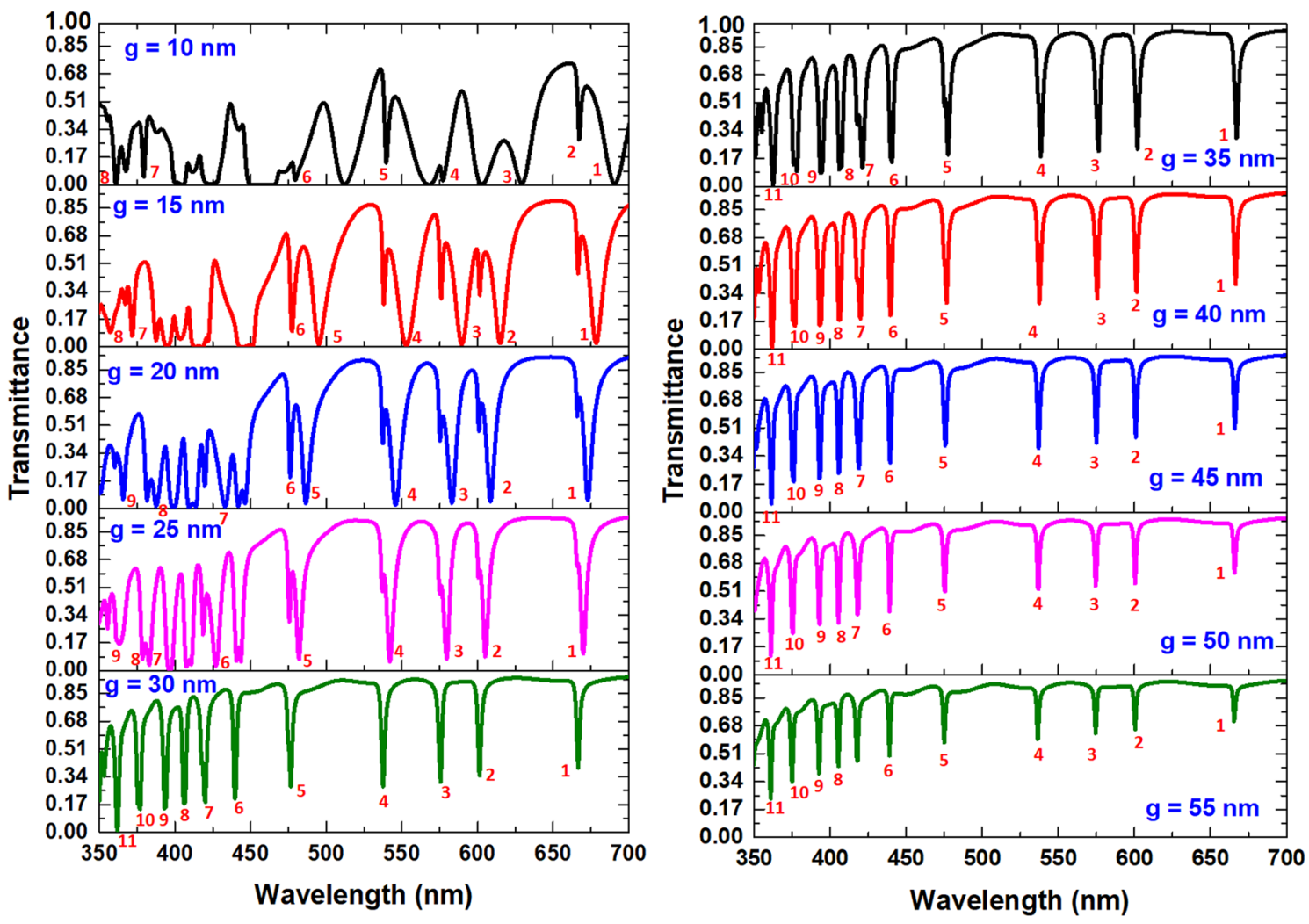

Figure 4. Transmittance spectrum of the proposed color filter for different values of $g$ from 10 to $55 \mathrm{~nm}$, with an interval of $5 \mathrm{~nm}$.

SPPs wave hardly stays in the six cavities, showing that the SPPs wave in the bus waveguide has a constructive interference, while in the cavity has a destructive interference.

On the contrary, plasmon modes are restricted predominantly to the left side of the bus waveguide and the cavities at $\lambda_{\text {res }}$. As a result, SPPs cannot propagate on the right side of the bus waveguide, which accords with the observation of low transmittance (i.e., long $\Delta \mathrm{D}$ ) at $\lambda_{\text {res }}$. Based on the simulations (not shown here for simplicity), the proposed structure cannot form multiple channels and filter functions if the proposed system only exists in one cavity. As observed in Fig. 3b-l, different resonance modes confine in the specific cavity, which offers eleven channels of $\lambda_{\text {res }}$, respectively. Thus, the circular cavities can behave as the Fabry-Pérot cavities in the optical filter system, and one of six cavities can effectively trap the incident light at $\lambda_{\text {res }}$. According to the $|E|$ field patterns, the SPPs wave can couple well because of the constructive interference between the bus waveguide and one of the six circular cavity resonators, exhibiting a significant CPR effect. The |E| field enhancement of the SPPs modes in the six circular cavities reveals an excellent light-matter interaction. Besides, the dipole effect, i.e., positive-negative charge pairs, may induce along the periphery of the six circular cavities, forming the vigorous confinement of SPPs and providing destructive interference at the right end of the bus waveguide. Thus, the evident transmittance dips could attain in Fig. 2.

\section{Optimization of the parameters of $g$ and $d$}

The SPPs modes originating from the proposed color filter are due to the coupling effect between the bus waveguide and the six circular cavities, significantly influenced by the structural size. For ease of fabrication, the structural parameters are less as possible. In the proposed color filter, $\mathrm{w}$ is fixed at $100 \mathrm{~nm}$ to promise that the TM mode can propagate in the bus waveguide. According to the FEM simulations (not shown here for simplicity), we can get the different colors of $\lambda_{\text {res }}$ by modifying suitable radii of circular cavities (i.e., $r_{1}-r_{6}$ ) based on the structural parameters listed in Table 1 . In other words, we can attain the desired color in terms of wavelength ranging in ultraviolet and visible by choosing an appropriate size of circular cavities. Therefore, we further examine the other two parameters in our simulations, i.e., $g$ and $d$.

First, Fig. 4 depicts the transmittance spectrum of the proposed color filter for different values of $g$ from 10 to $55 \mathrm{~nm}$, with an interval of $5 \mathrm{~nm}$. The other structural parameters are the same as used in Table 1 . We numbered the available operation channels depending on the sharper resonance dip, smaller FWHM, and more extended $\Delta \mathrm{D}$ in the inset of this figure. As seen, the transmittance dips decrease with the increasing $g$. The transmittance has a different line shape to the variation of $g$ because of their different coupling effect between bus waveguide and side-coupled cavities. Each channel has a slight resonance dip at the left side of the saddle point when $g \leq 25 \mathrm{~nm}$ and vanishes when $g \geq 30 \mathrm{~nm}$. Furthermore, when $\mathrm{g} \leq 25 \mathrm{~nm}$, the resonance dips are not sharp enough in the short wavelength range (i.e., ranging in $350-450 \mathrm{~nm}$ ). It is worth noting that the transmittance curves show a 

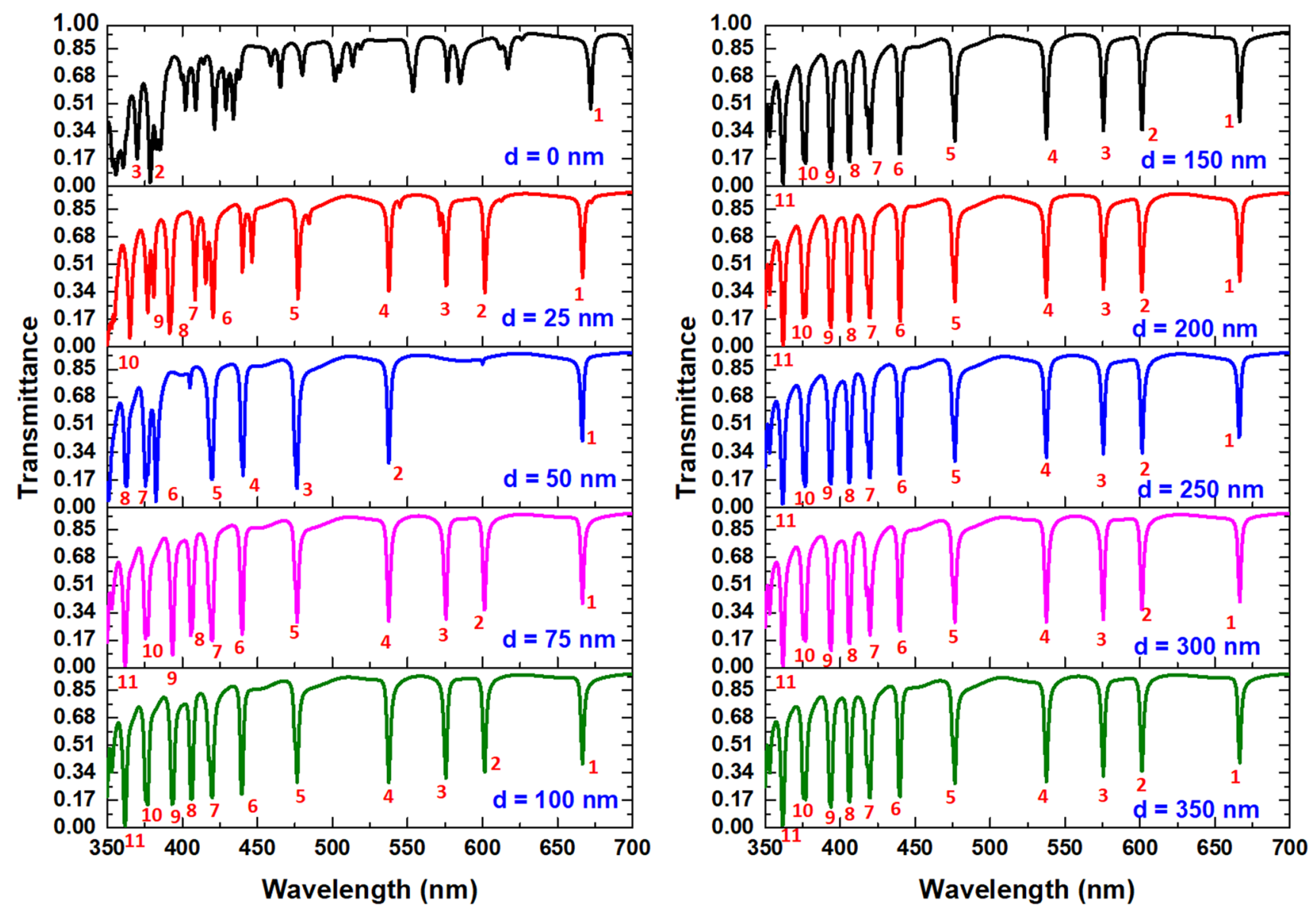

Figure 5. Transmission spectra as a function of $d$ variation of the proposed color filter.

strong oscillation since the more substantial coupling effect is when $g \leq 25 \mathrm{~nm}$. Besides, the $\Delta \mathrm{D}$ and FWHM will significantly reduce when $g \geq 50 \mathrm{~nm}$ due to the more negligible coupling effect of a more significant $g$. Based on Fig. 4, the acceptable values of $g$ are in the range of $25-45 \mathrm{~nm}$ based on the viewpoint of the workable channel numbers, transmittance line-shape, $\Delta \mathrm{D}$, and FWHM.

Successively, we show the variation influence of $d$ on the transmittance spectrum in Fig. 5. As shown, a short $\Delta \mathrm{D}$ and a fierce oscillation of transmittance line-shape arising from the violent coupling effect between the connected cavities when $d=0 \mathrm{~nm}$. Nevertheless, the $\lambda_{\text {res }}$ remains the same values with increasing $d$ since the less interaction between adjacent holes when $d \geq 25 \mathrm{~nm}$. In general, a mistake during fabrication has a severe influence on the efficacy of plasmonic devices. A slight error in different structural sizes can significantly alter the sensitivity performance. Fortunately, the shift of $\lambda_{\text {res }}$ by varying $d$ is less sensitive to the change of $d$, implying that the robust fabrication of the proposed color filter. Based on Fig. 5, the workable values of $d$ can choose in the range of $25-350 \mathrm{~nm}$.

\section{Application as a refractive index sensor}

The proposed color filter can function as a RI sensor when the structure involves the detecting medium, i.e., the $n_{\text {eff }}$ in the bus waveguide and six circular cavities is changed and is exceptionally responsive to the surrounding material. Figure 6 shows the transmittance spectra of the proposed color filter with the ambient media, $n$, are $1.01,1.02$, and 1.03, respectively. As observed, the transmission dips show a redshift as the increasing RI and a linear relationship between the $n_{\text {eff }}$ and the $\lambda_{\text {res }}$, which well agrees with Eq. (2). The sensitivity's increment is closely related to the SPR and CPR effects in the color filter, leading to an interaction with the variation of the surrounding medium ${ }^{67}$.

An excellent RI sensor should have the features of the high value of sensitivity (S), figure of merit (FOM), Q-factor, and dipping strength $(\Delta \mathrm{D})$. Figure 7 depicts the calculated $\lambda_{\text {res }}$ from mode 1 to mode 11 for the proposed color filter versus the RI with the surrounding media, $n$, in the range of 1.01-1.05 with an increment of 0.01 . We outline the $\mathrm{S}, \mathrm{FOM}, \mathrm{Q}$ factor, and $\Delta \mathrm{D}$ of the proposed color filter from mode 1 to mode 11 in Table 2. Note that the maximum values of $\mathrm{S}, \mathrm{FOM}, \mathrm{Q}$ factor, and $\triangle \mathrm{D}$ obtained from mode 1 to mode 11 can achieve $700 \mathrm{~nm} / \mathrm{RIU}, 350$ 1/RIU, 349.0, and 65.04\%, respectively, reveal distinguished sensor properties of the proposed color filter. These values are more remarkable than the previous articles (e.g., ${ }^{37,42}$ ) and show multiple channels of the color filter and RI sensor functions that can match the requisite wavelength of ultraviolet and visible. We compare the designed structure with the published similar systems in Table 3. Besides, we can vary the desired 


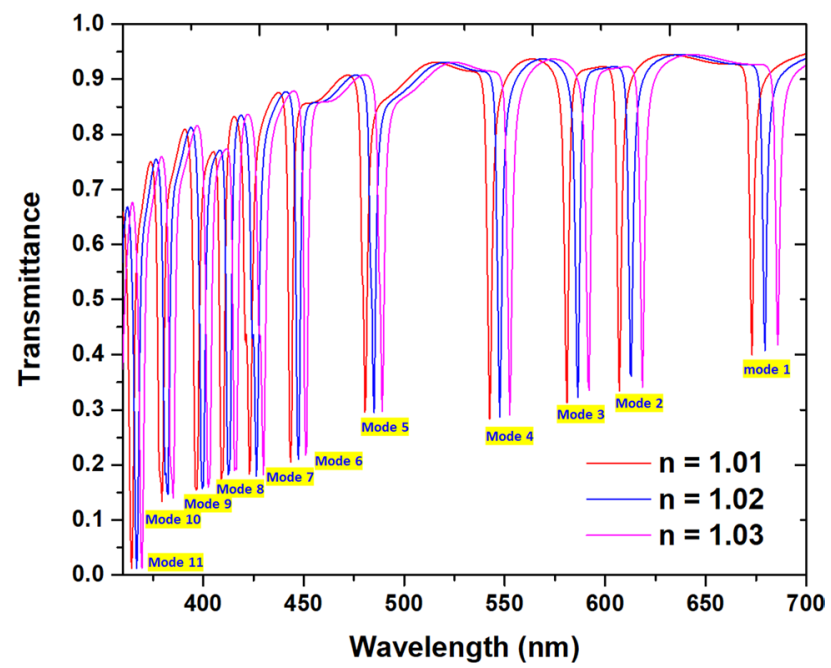

Figure 6. Transmittance spectra of eleven channels (i.e., modes 1-11) in the proposed color filter with the ambient media, $n$, are $1.01,1.02$, and 1.03 , respectively.

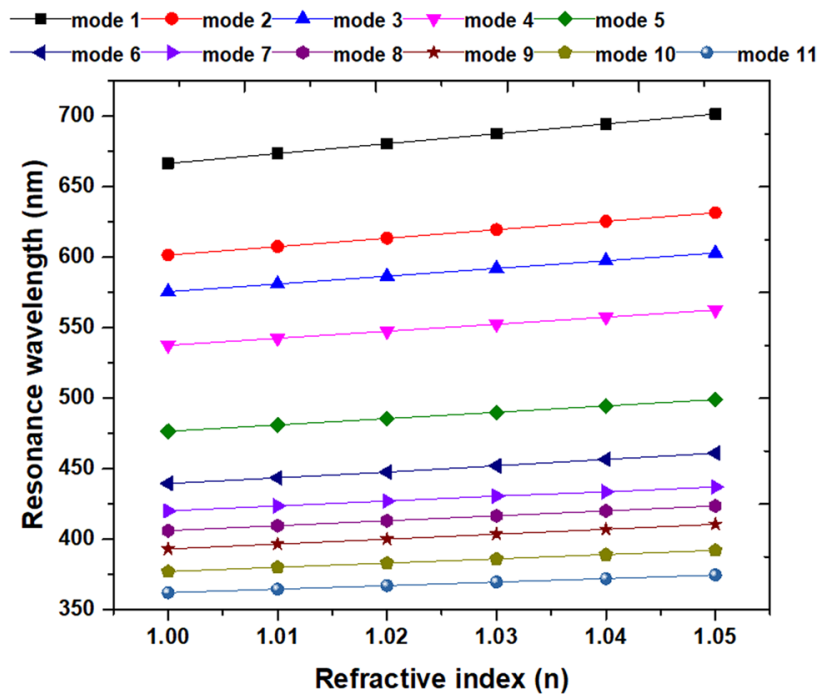

Figure 7. Calculated resonance wavelength $\left(\lambda_{\text {res }}\right)$ from mode 1 to mode 11 for the proposed color filter versus the RI with the filling media, $n$, in the range of $1.01-1.05$ in the step of 0.01 .

\begin{tabular}{|l|l|l|l|l|l|l|l|l|l|l|l|}
\hline Mode & $\mathbf{1}$ & $\mathbf{2}$ & $\mathbf{3}$ & $\mathbf{4}$ & $\mathbf{5}$ & $\mathbf{6}$ & $\mathbf{7}$ & $\mathbf{8}$ & $\mathbf{9}$ & $\mathbf{1 0}$ & $\mathbf{1 1}$ \\
\hline $\mathrm{S}(\mathrm{nm} / \mathrm{RIU})$ & 700 & 600 & 550 & 500 & 450 & 400 & 350 & 350 & 350 & 300 & 250 \\
\hline FOM $(/ \mathrm{RIU})$ & 350.0 & 300.0 & 275.0 & 250.0 & 225.0 & 200.0 & 175.0 & 175.0 & 175.0 & 150.0 & 125.0 \\
\hline Q factor & 349.0 & 315.0 & 301.2 & 281.3 & 248.6 & 229.5 & 218.5 & 211.0 & 204.6 & 195.3 & 187.3 \\
\hline$\Delta \mathrm{D}(\%)$ & 53.28 & 57.66 & 63.00 & 65.01 & 62.54 & 66.71 & 64.85 & 58.70 & 65.89 & 60.39 & 65.04 \\
\hline
\end{tabular}

Table 2. The S, FOM, Q factor, and $\Delta \mathrm{D}$ of the proposed color filter from modes 1 to 11 .

working wavelengths by optimization of structural parameters. The other RI values has the same trend as the obtained results from Fig. 7 . 


\begin{tabular}{|l|l|l|l|l|c|}
\hline References & Number of channels & Max. S (nm/RIU) & FOM (1/RIU) & $\Delta$ D & FWHM (nm) \\
\hline 37 & 6 & 608 & 57.53 & 85.00 & 10.56 \\
\hline 68 & 1 & - & 16.70 & 70.00 & 76.00 \\
\hline 69 & 9 & - & 156.94 & 30.00 & 150.00 \\
\hline 38 & 3 & - & - & 40.00 & 150.00 \\
\hline 42 & 6 & 700 & 191.6 & 61.00 & 40.00 \\
\hline 29 & 1 & - & 159.6 & 65.00 & 15.00 \\
\hline 70 & 4 & - & - & 40.00 & 60.00 \\
\hline This work & 11 & 700 & 255.56 & 81.89 & 2.00 \\
\hline
\end{tabular}

Table 3. Comparison of the proposed optical filter with the previous similar color filter structures.

\begin{tabular}{|l|l|l|l|l|l|l|l|l|l|l|l|}
\hline Mode & $\mathbf{1}$ & $\mathbf{2}$ & $\mathbf{3}$ & $\mathbf{4}$ & $\mathbf{5}$ & $\mathbf{6}$ & $\mathbf{7}$ & $\mathbf{8}$ & $\mathbf{9}$ & $\mathbf{1 0}$ & $\mathbf{1 1}$ \\
\hline $\mathrm{M}_{\mathrm{ext}}(\mathrm{dB})$ & 37.09 & 42.53 & 48.46 & 50.82 & 50.79 & 62.48 & 65.17 & 63.25 & 73.97 & 72.05 & 174.50 \\
\hline
\end{tabular}

Table 4. The extinction ratio values of the proposed color filter from mode 1 to 11.

\section{Application as an optical switch}

Optical switching is a vital device for applying in IOCs ${ }^{71,72}$. Recently, some all-optical switches based on MIM waveguides have been developed and investigated since the features of strong light confinement and electromagnetically induced transparency (EIT) spectra ${ }^{73-75}$. This kind of all-optical switch could overcome diffraction limits in nano-scale dimensions. Liu et al. proposed an ultrafast and low-power all-optical switch with femtosecondscale feedback time using a MIM waveguide with a slot ${ }^{73}$. Negahdari et al. designed an all-optical plasmonic switch based on a MIM split square ring resonator with the reverse and direct switching operations ${ }^{74}$. Bazgir et al. used MIM-based dumbbell-shaped cavity slots by exploiting a DNA composite and claimed the switchable DNA element can manipulate the electric field and transmission line shape ${ }^{75}$. The sharp transmittance line shapes with high FOM, Q-factor, and dipping strength obtained in the proposed color filter are workable for constructing optical switches. In Fig. 2, the transmittance curve displays a steep decline from transmittance peak ("on") to transmittance dip ("off”) with a small FWHM, and thus can utilize this property to establish an optical switch device. The transmission contrast ratio can express as ${ }^{76}$

$$
\mathrm{M}_{\mathrm{ext}}=10 \log \left(\mathrm{T}_{\max } / \mathrm{T}_{\min }\right)(\mathrm{dB})
$$

where $\mathrm{T}_{\max }$ and $\mathrm{T}_{\min }$ represent "on" (peak) and "off" (dip), respectively. Based on Eq. (4), we have a maximum extinction ratio of $174.50 \mathrm{~dB}$ and summarize the extinction ratio values of the proposed color filter in Table 4 . These values are great higher than the previous literature in the wavelength range of ultraviolet and visible (e.g., $\left.{ }^{71,76-78}\right)$.

\section{Application for inspecting hemoglobin concentration in human blood samples}

The proposed color filter can also detect biological fluids, e.g., hemoglobin concentration (HC), since its suitable wavelength range with multiple resonance modes and excellent sensing performance ${ }^{79}$. For application, six cavities and the bus waveguide can be used as the resonator and then the blood specimen placed inside them. In a practical situation, the blood sample can drop into the air regions of six circular-shape cavities by capillary attraction $^{80-82}$, and the bus waveguide with a small amount due to the SPPs are very sensitive to the variation of the ambient material ${ }^{83,84}$. Several research groups experimentally and theoretically investigated the RI of blood groups $(\mathrm{O}, \mathrm{A}$, and $\mathrm{B})$ at visible and near-infrared wavelengths $(380-1100 \mathrm{~nm})$ for diverse blood specimens ${ }^{85-87}$, in which the operation wavelengths quite fit our proposed color filter.

The relationship between the blood samples' RI and HC can signify by ${ }^{88}$

$$
n_{b}=n_{0}+\alpha C
$$

where $n_{0}$ is the blood sample's effective RI when $\mathrm{C}=0$. $C$ is the blood sample's HC (in $\mathrm{g} / \mathrm{L}$ ), and $\alpha$ is the specific refraction increment fixes a particular blood group. Besides, the temperature $\mathrm{T}$ (in the Kelvin unit) and the operating wavelength can also influence the value of $n_{b}$. As a result, Eq. (5) can express by ${ }^{81,84}$ :

$$
n_{b}=n_{0}+\alpha C+\beta T+\delta \lambda+\sigma \lambda^{2}+\gamma \lambda^{3},
$$

where $\alpha, \beta, \delta, \sigma, \gamma$ are Cauchy coefficients that vary with blood group, and these coefficients can obtain from Ref. ${ }^{80}$.

Based on the calculation results from Eq. (6), the $\mathrm{HC}$ of different blood group specimens in Ref. ${ }^{89}$ for temperature at $\mathrm{T}=300^{\circ} \mathrm{K}$ is found as $\mathrm{C}_{\mathrm{A}}=146.2 \mathrm{~g} / \mathrm{L}, \mathrm{C}_{\mathrm{B}}=111.1 \mathrm{~g} / \mathrm{L}$ and $\mathrm{C}_{\mathrm{O}}=108.9 \mathrm{~g} / \mathrm{L}$. Figure 8 depicts the transmittance spectrum of three different blood groups $(\mathrm{O}, \mathrm{A}$, and $\mathrm{B})$ for $\mathrm{C}_{\mathrm{A}}=146.2 \mathrm{~g} / \mathrm{L}, \mathrm{C}_{\mathrm{B}}=111.1 \mathrm{~g} / \mathrm{L}$, and $\mathrm{C}_{\mathrm{O}}=108.9 \mathrm{~g} / \mathrm{L}$ in room temperature $\left(300{ }^{\circ} \mathrm{K}\right)$ put in the proposed color filter. The geometrical parameters are the same as used in Table 1. As shown in Fig. 8, we have thirteen channels (labeled by number) in the 500-1120 nm wavelength. These 


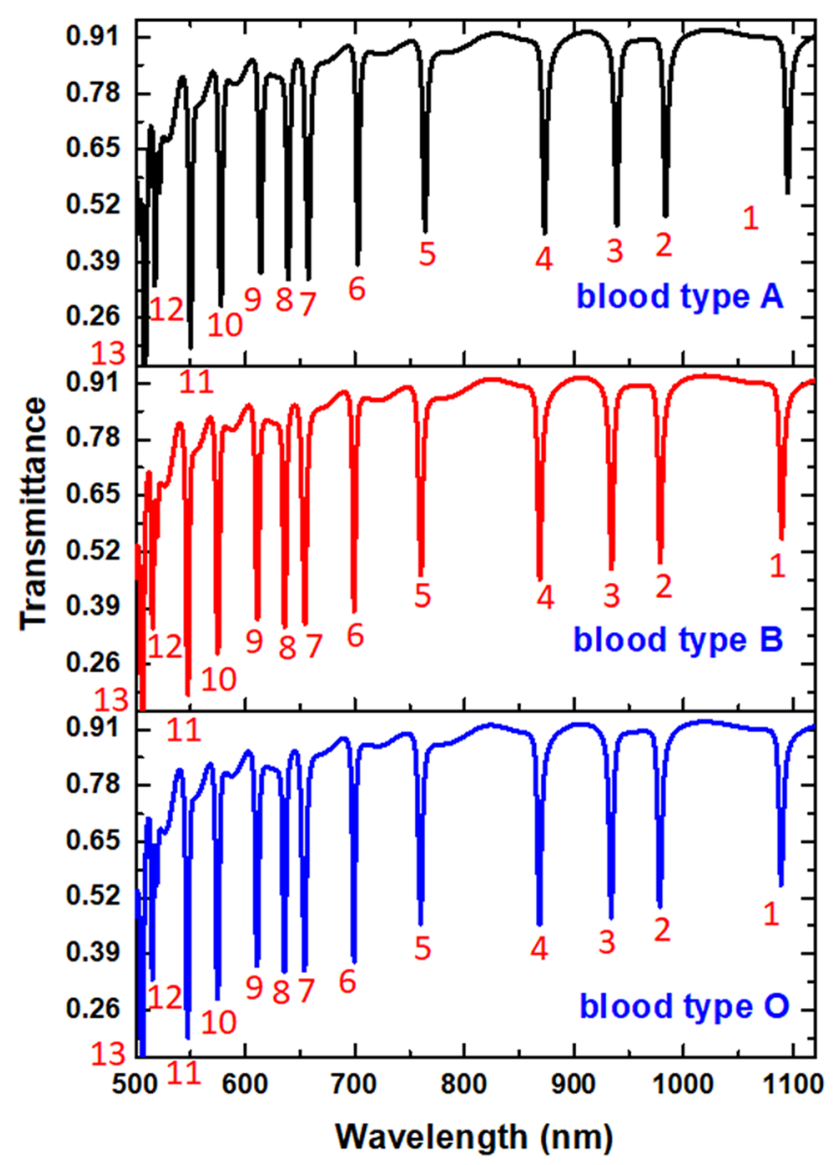

Figure 8. Transmittance spectrum of three different blood groups $(\mathrm{O}, \mathrm{A}$, and $\mathrm{B})$ for $\mathrm{CA}=146.2 \mathrm{~g} / \mathrm{L}$, $\mathrm{CB}=111.1 \mathrm{~g} / \mathrm{L}$, and $\mathrm{CO}=108.9 \mathrm{~g} / \mathrm{L}$ at $300^{\circ} \mathrm{K}$ put in the proposed color filter.

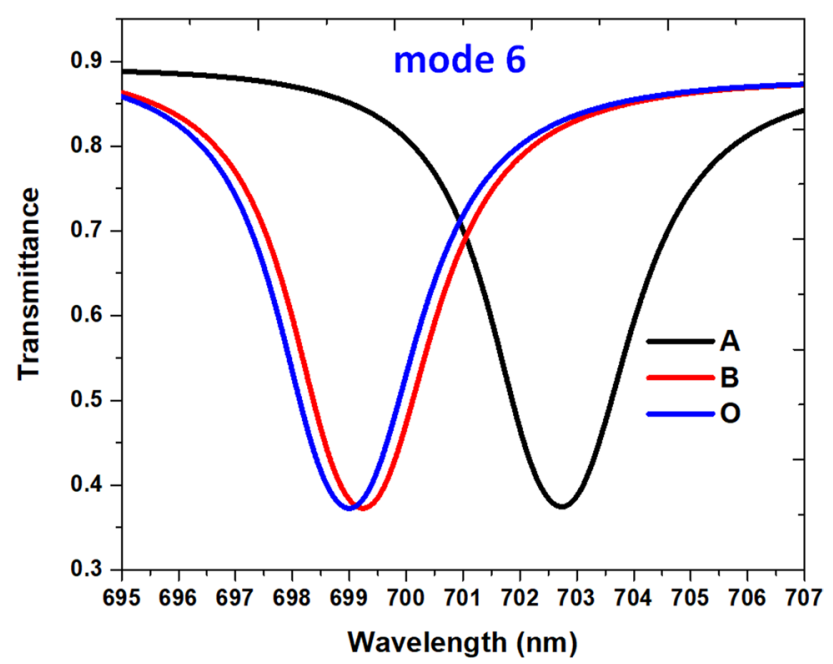

Figure 9. Illustration of the transmittance spectrum of mode 6 in the wavelength of 695-707 nm.

ranges can shift from the ultraviolet and visible light spectra blend into the near-infrared range. Compared to its counterpart without solution (see Fig. 2), the two more working channels redshift from the shorter wavelengths because of the increase of the $n_{\text {eff }}$ of HC specimens (see Eq. (2)). Using the recently introduced detectors can achieve a $\lambda_{\text {res }}$ shift of as small as $0.1 \mathrm{~nm}$. We illustrate the transmittance spectrum of mode 6 in the wavelength of 695-707 $\mathrm{nm}$ as an example (see Fig. 9). The $\lambda_{\text {res }}$ for A, B, and O-groups are $702.74 \mathrm{~nm}, 699.24 \mathrm{~nm}$, and $699.00 \mathrm{~nm}$, respectively. Note that a very narrow FWHM $(\sim 2 \mathrm{~nm})$ is attainable in each curve. 

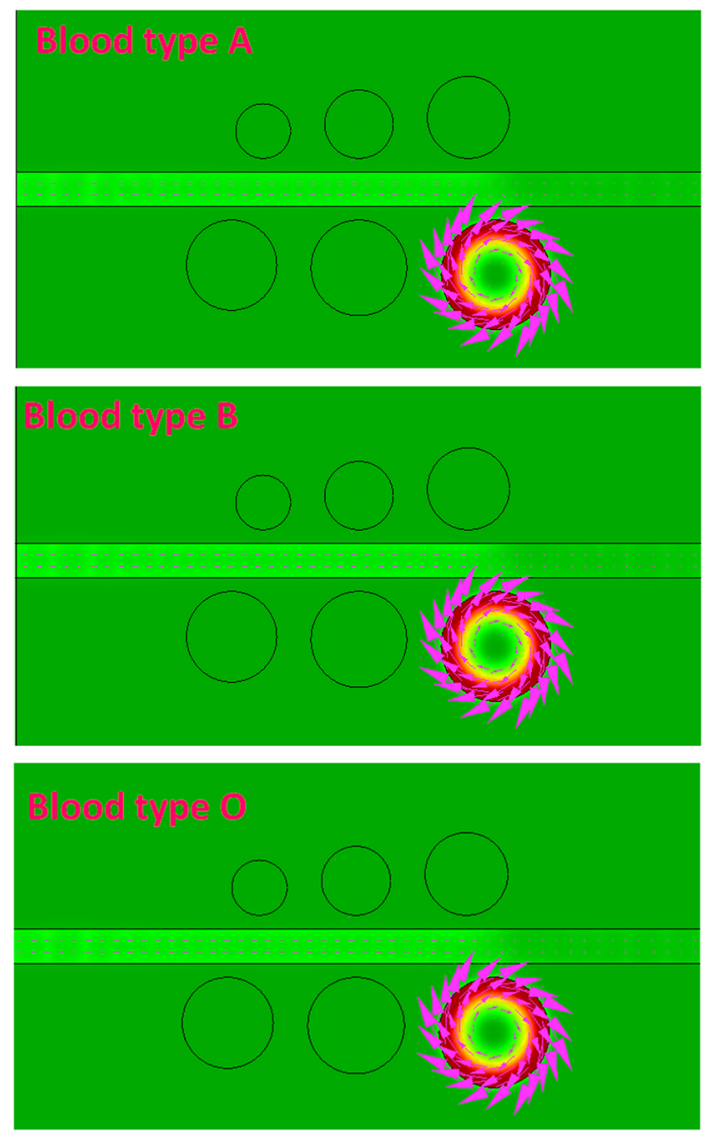

$\mathrm{W} / \mathrm{m}^{2}$

0

$1 \times 10^{8}$

Figure 10. Time average power flow $\left(\mathrm{W} / \mathrm{m}^{2}\right)$ with arrows of three blood types of hemoglobin concentrations, i.e., $146.2 \mathrm{~g} / \mathrm{L}, 111.1 \mathrm{~g} / \mathrm{L}$, and $108.9 \mathrm{~g} / \mathrm{L}$, for mode 6 .

Going further into their mechanism, Fig. 10 shows the time average power flow $\left(\mathrm{W} / \mathrm{m}^{2}\right)$ with arrows of three blood types of HCs, i.e., $146.2 \mathrm{~g} / \mathrm{L}, 111.1 \mathrm{~g} / \mathrm{L}$, and $108.9 \mathrm{~g} / \mathrm{L}$, for mode 6 . As observed, the power flows well tripped in the right side of the bottom cavities. Significantly, the high-density energy flows appear in the interface between the bus waveguide and resonators. Besides, the dense energy flows propagate on the cavity's circumference surface and efficiently connect to the bus waveguide, showing a better coupling effect between the bus waveguide and cavities. As the three blood types of HCs are involved in the plasmonic filter system, leading to increased local environmental RI, the $\lambda_{\text {res }}$ will redshift since the reduced repulsion between dipoles (i.e., positive-negative pairs) with the same orientation, thus reducing the energy of the plasmon oscillations. It is important to note that most of the power flows in each case formed a ring-like spot on the cavity's periphery surface and propagated in a clockwise direction. These results show that the measurement of HCs with the proposed color filter can be conducted conveniently with high accuracy at the desired wavelength, and the proposed design can apply in diverse biomedical and RI sensing applications.

According to Eq. (6), parameters such as $\mathrm{HC}$ and temperature closely relate to the RI variation of different blood groups. In addition, oxygenated hemoglobin has a more negligible light absorbance at $\lambda=700 \mathrm{~nm}$ than deoxygenated hemoglobin; thus, we examined the incident wavelength of $\lambda=700 \mathrm{~nm}$. Figure 11a plots the RI variations corresponding to the three blood types within the $\mathrm{HC}$ ranging in $90-180 \mathrm{~g} / \mathrm{L}$ for a selected working wavelength $(\lambda=700 \mathrm{~nm})$ at the temperature of $T=300 \mathrm{~K}$. As seen, the RI of the three blood types increases linearly with the increase of HC. As a result, one can get the related HC if the RI can attain through the SPPs modes inspection. Figure $11 \mathrm{~b}$ also reveals the varying RI with temperature $\mathrm{T}$ in the range of $260-350{ }^{\circ} \mathrm{K}$ at $\lambda=700 \mathrm{~nm}$ when the HCs chose $C_{A}=146.2 \mathrm{~g} / \mathrm{L}, \mathrm{C}_{\mathrm{B}}=111.1 \mathrm{~g} / \mathrm{L}$ and $\mathrm{C}_{\mathrm{O}}=108.9 \mathrm{~g} / \mathrm{L}$, respectively. In Eq. (6), the parameter $\mathrm{T}$ (temperature) closely relates to the RI variation of different blood groups, and $\mathrm{T}$ has less influence on the dimensions of the circular cavities and the air gap in the proposed MIM waveguide system ${ }^{80-82}$. As seen in Fig. 11b, the growth trend of the line profiles of RI versus $\mathrm{T}$ also displays a linear relationship between RI and $\mathrm{T}$, and as the $\mathrm{T}$ increases, the RI decreases. Therefore, the transmittance dip (i.e., $\lambda_{\text {res }}$ ) will blueshift as $\mathrm{T}$ increases because of the decrease of effective refractive index (RI) (see Eq. (2)). Compared with other reported devices for HC detection, the performance of our proposed color filter possesses novel features and flexible applications in the 


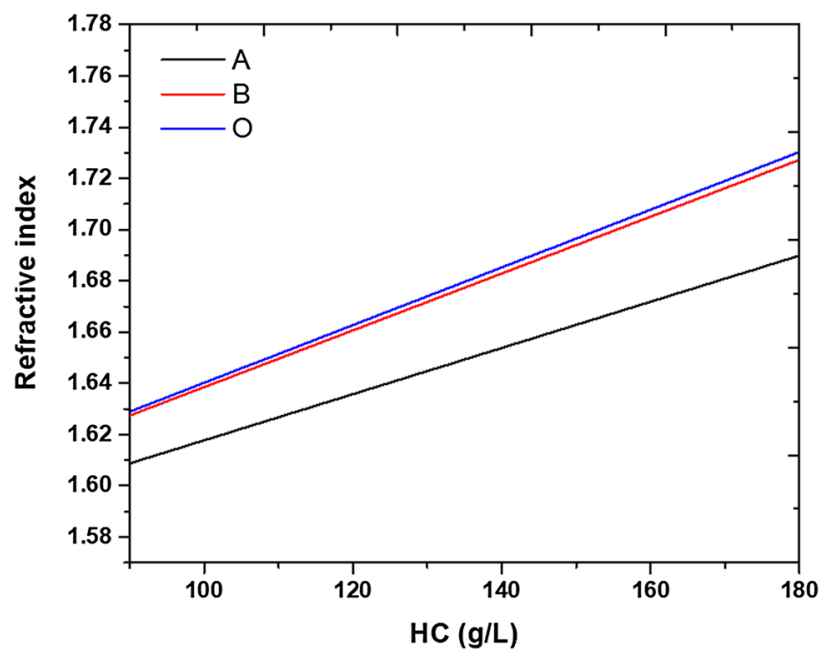

(a)

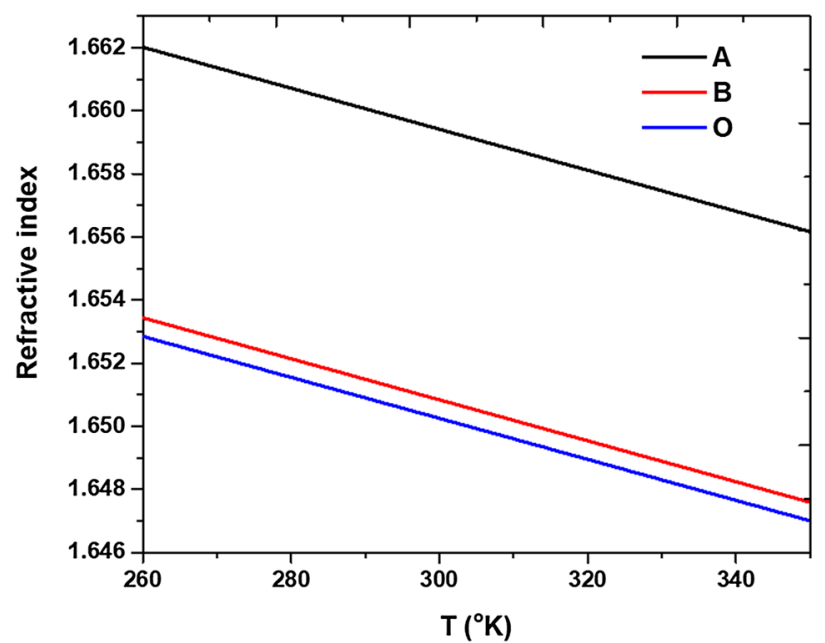

(b)

Figure 11. (a) Three blood types of refractive indices spectra versus hemoglobin concentration ranging in $90-180 \mathrm{~g} / \mathrm{L}$ for a selected working wavelength, $\lambda=700 \mathrm{~nm}$, at $\mathrm{T}=300{ }^{\circ} \mathrm{K}$. (b) Three blood types of refractive indices spectra versus the temperature in the range of $270-330{ }^{\circ} \mathrm{K}$ with $\mathrm{C}_{\mathrm{A}}=146.2 \mathrm{~g} / \mathrm{L}, \mathrm{C}_{\mathrm{B}}=111.1 \mathrm{~g} / \mathrm{L}$, and $\mathrm{C}_{\mathrm{O}}=108.9 \mathrm{~g} / \mathrm{L}$ for $\lambda=700 \mathrm{~nm}$.

wavelength range of 350-1100 $\mathrm{nm}$ and has the advantage of multiple channels and the functions of RI sensors and optical switches.

\section{Conclusion}

In summary, we propose a multichannel color filter capable of filtering out eleven modes, including individual colors in red, orange, yellow, green, blue, four violets, and two ultraviolets in the wavelength range of 350-700 nm. The designed structure consists of one MIM bus waveguide side-coupled to six circular cavities. We performed the simulations by using FEM to investigate the transmittance spectrum and EM wave distributions in detail. Such filter possesses the other two functions, including RI sensor and optical switch, meantime has an ultracompact scheme, less structural parameters, and small footprint, benefiting from the independent tunability of different colors. The maximum values of sensitivity, figure of merit, quality factor, dipping strength, and extinction ratio can reach $700 \mathrm{~nm} / \mathrm{RIU}, 350$ 1/RIU, 349.0, 65.04\%, and $174.50 \mathrm{~dB}$, respectively, which reveal the excellent functions of sensor performance and optical switch. This sensor can widely be used in gas and biochemistry since its ease of preparation, excellent sensing performance, and broad working wavelengths with multiple modes, suitable for detecting gases and fluids (e.g., hemoglobin concentration). This research can receive pivotal applications on highly IOCs.

\section{Data availability}

The authors declare that all data supporting the findings of this study are available from the corresponding author upon reasonable request. 
Received: 29 September 2021; Accepted: 10 November 2021

Published online: 25 November 2021

\section{References}

1. Wang, K. et al. Plasmon-enhanced optical nonlinearity for femtosecond all-optical switching. Appl. Phys. Lett. 111, 181102. https:// doi.org/10.1063/1.5002581 (2017).

2. Chau, Y.-F. et al. Localized resonance of composite core-shell nanospheres, nanobars and nanospherical chains. Prog. Electromagn. Res. B 28, 183-199 (2011).

3. Chen, M. W., Chau, Y.-F. \& Tsai, D. P. Three-dimensional analysis of scattering field interactions and surface plasmon resonance in coupled silver nanospheres. Plasmonics 3, 157. https://doi.org/10.1007/s11468-008-9069-8 (2008).

4. Chau, Y.-F. Surface plasmon effects excited by the dielectric hole in a silver-shell nanospherical pair. Plasmonics 4, 253. https://doi. org/10.1007/s11468-009-9100-8 (2009).

5. Ho, Y. Z. et al. Tunable plasmonic resonance arising from broken-symmetric silver nanobeads with dielectric cores. J. Opt. 14, 114010. https://doi.org/10.1088/2040-8978/14/11/114010 (2012).

6. Sung, M.-J., Ma, Y.-F., Chau, Y.-F. \& Huang, D.-W. Surface plasmon resonance in a hexagonal nanostructure formed by seven core shell nanocylinders. Appl. Opt. 49, 920-926. https://doi.org/10.1364/AO.49.000920 (2010).

7. Peng, T.-C., Lin, W.-C., Chen, C.-W., Tsai, D. P. \& Chiang, H.-P. Enhanced sensitivity of surface plasmon resonance phaseinterrogation biosensor by using silver nanoparticles. Plasmonics 6, 29-34. https://doi.org/10.1007/s11468-010-9165-4 (2011).

8. Shen, L., Yang, T.-J. \& Chau, Y.-F. Effect of internal period on the optical dispersion of indefinite-medium materials. Phys. Rev. B 77, 205124. https://doi.org/10.1103/PhysRevB.77.205124 (2008).

9. Lin, C.-T. et al. Rapid fabrication of three-dimensional gold dendritic nanoforests for visible light-enhanced methanol oxidation. Electrochim. Acta https://doi.org/10.1016/j.electacta.2016.01.043 (2016).

10. Chau, Y.-F. \& Tsai, D. P. Three-dimensional analysis of silver nano-particles doping effects on super resolution near-field structure. Opt. Commun. 269, 389-394. https://doi.org/10.1016/j.optcom.2006.08.004 (2007).

11. Shen, L., Yang, T.-J. \& Chau, Y.-F. 50/50 beam splitter using a one-dimensional metal photonic crystal with parabolalike dispersion. Appl. Phys. Lett. 90, 251909. https://doi.org/10.1063/1.2750385 (2007).

12. Zhang, J. \& Zhang, L. Nanostructures for surface plasmons. Adv. Opt. Photon. 4, 157-321. https://doi.org/10.1364/AOP.4.000157 (2012).

13. Singh, L., Maccaferri, N., Garoli, D. \& Gorodetski, Y. Directional plasmonic excitation by helical nanotips. Nanomaterials 11, 1333 (2021).

14. Zhu, J., Wang, G., Jiang, F., Qin, Y. \& Cong, H. Temperature sensor of MoS2 based on hybrid plasmonic waveguides. Plasmonics 14, 1863-1870. https://doi.org/10.1007/s11468-019-00988-9 (2019).

15. Hsieh, L.-Z. et al. Metal nano-particles sizing by thermal annealing for the enhancement of surface plasmon effects in thin-film solar cells application. Opt. Commun. 370, 85-90. https://doi.org/10.1016/j.optcom.2016.03.009 (2016).

16. Wu, P., Zhang, C., Tang, Y., Liu, B. \& Lv, L. A perfect absorber based on similar Fabry-Perot four-band in the visible range. Nanomaterials 10, $488(2020)$.

17. Chou Chau, Y.-F. et al. Perfect dual-band absorber based on plasmonic effect with the cross-hair/nanorod combination. Nanomaterials 10, 2030. https://doi.org/10.3390/nano10030493 (2020).

18. Wei, Q., Xiao, J., Yang, D. \& Cai, K. Ultra-compact electro-optic modulator based on alternative plasmonicmaterial. Appl. Opt. 60, 5252-5257. https://doi.org/10.1364/AO.425679 (2021).

19. Ansell, D. et al. Hybrid graphene plasmonic waveguide modulators. Nat. Commun. 6, 8846. https://doi.org/10.1038/ncomms9846 (2015).

20. Fang, C. \& Xu, Y. Tunable power splitter based on MIM waveguide-rectangle cavity system with Kerr material. Mod. Phys. Lett. B 30, 1650376. https://doi.org/10.1142/S0217984916503760 (2016).

21. Zhang, T. \& Shan, F. Development and application of surface plasmon polaritons on optical amplification. J. Nanomater. 2014, 495381. https://doi.org/10.1155/2014/495381 (2014).

22. Izadi, M. A. \& Nouroozi, R. Adjustable propagation length enhancement of the surface plasmon polariton wave via phase sensitive optical parametric amplification. Sci. Rep. 8, 15495. https://doi.org/10.1038/s41598-018-33831-y (2018).

23. Sahu, P. P. Optical switch based on graphene clad two surface plasmonic polariton mode coupler. Optik 227, 166026. https://doi. org/10.1016/j.ijleo.2020.166026 (2021).

24. Bashiri, S. \& Fasihi, K. An all-optical $1 \times 2$ demultiplexer using Kerr nonlinear nano-plasmonic switches. Plasmonics 15, $449-456$. https://doi.org/10.1007/s11468-019-01068-8 (2020).

25. Neutens, P., Lagae, L., Borghs, G. \& Van Dorpe, P. Plasmon filter and resonator in metal-insulator-metal waveguides. Opt. Express 20, 3408-3423. https://doi.org/10.1364/OE.20.003408 (2012).

26. Lin, J.-M. \& Chau, Y.-F. Radome slope compensation using multiple-model Kalman filters. J. Guid. Control Dyn. 18, 637-640. https://doi.org/10.2514/3.21438 (1995).

27. Zhu, J. \& Li, N. MIM waveguide structure consisting of a semicircular resonant cavity coupled with a key-shaped resonant cavity. Opt. Express 28, 19978-19987. https://doi.org/10.1364/OE.395696 (2020).

28. Chen, Y. et al. Sensing performance analysis on Fano resonance of metallic double-baffle contained MDM waveguide coupled ring resonator. Opt. Laser Technol. 101, 273-278. https://doi.org/10.1016/j.optlastec.2017.11.022 (2018).

29. Rahmatiyar, M., Afsahi, M. \& Danaie, M. Design of a refractive index plasmonic sensor based on a ring resonator coupled to a mim waveguide containing tapered defects. Plasmonics 15, 2169-2176. https://doi.org/10.1007/s11468-020-01238-z (2020).

30. Wen, K. et al. Fano resonance with ultra-high figure of merits based on plasmonic metal-insulator-metal waveguide. Plasmonics 10, 27-32. https://doi.org/10.1007/s11468-014-9772-6 (2014).

31. Zhang, Z. D., Wang, H. Y. \& Zhang, Z. Y. Fano resonance in a gear-shaped nanocavity of the metal-insulator-metal waveguide. Plasmonics 8, 797-801. https://doi.org/10.1007/s11468-012-9475-9 (2013).

32. Jankovic, N. \& Cselyuszka, N. Multiple Fano-like MIM plasmonic structure based on triangular resonator for refractive index sensing. Sensors 18, 287 (2018).

33. Zhu, J. \& Wu, C. Optical refractive index sensor with Fano resonance based on original MIM waveguide structure. Results Phys. 21, 103858. https://doi.org/10.1016/j.rinp.2021.103858 (2021).

34. Sagor, R. H., Hassan, M. F., Sharmin, S., Adry, T. Z. \& Emon, M. A. R. Numerical investigation of an optimized plasmonic on-chip refractive index sensor for temperature and blood group detection. Results Phys. 19, 103611. https://doi.org/10.1016/j.rinp.2020. 103611 (2020).

35. Chou Chau, Y.-F. et al. Significantly enhanced coupling effect and gap plasmon resonance in a MIM-cavity based sensing structure. Sci. Rep. 11, 18515. https://doi.org/10.1038/s41598-021-98001-z (2021).

36. Myland, P., Babilon, S. \& Khanh, T. Q. Tackling heterogeneous color registration: Binning color sensors. Sensors 21, 2950 (2021).

37. Zhang, Z. et al. A plasmonic ellipse resonator possessing hybrid modes for ultracompact chipscale application. Phys. Scr. 94, 125511. https://doi.org/10.1088/1402-4896/ab4677 (2019).

38. Diest, K., Dionne, J. A., Spain, M. \& Atwater, H. A. Tunable color filters based on metal-insulator-metal resonators. Nano Lett. 9, 2579-2583. https://doi.org/10.1021/nl900755b (2009). 
39. Lee, T., Jang, J., Jeong, H. \& Rho, J. Plasmonic- and dielectric-based structural coloring: from fundamentals to practical applications. Nano Convergence 5, 1. https://doi.org/10.1186/s40580-017-0133-y (2018).

40. Zayats, A., Smolyaninov, I. \& Maradudin, A. Nano-optics of surface plasmon polaritons. Phys. Rep. 4082011, 131-314. https:// doi.org/10.1016/j.physrep.2004.11.001 (2005).

41. Barnes, W. L., Dereux, A. \& Ebbesen, T. W. Surface plasmon subwavelength optics. Nature 424, 824-830. https://doi.org/10.1038/ nature 01937 (2003).

42. Butt, A., Khonina, S. \& Kazanskiy, N. A plasmonic colour filter and refractive index sensor applications based on metal-insulator-metal square $\mu$-ring cavities. Laser Phys. 30, 016205. https://doi.org/10.1088/1555-6611/ab5578 (2019).

43. El Haffar, R., Farkhsi, A. \& Mahboub, O. Optical properties of MIM plasmonic waveguide with an elliptical cavity resonator. Appl. Phys. A 126, 486. https://doi.org/10.1007/s00339-020-03660-w (2020).

44. Chu, Y., Schonbrun, E., Yang, T. \& Crozier, K. B. Experimental observation of narrow surface plasmon resonances in gold nanoparticle arrays. Appl. Phys. Lett. 93, 181108. https://doi.org/10.1063/1.3012365 (2008).

45. Bahramipanah, M., Abrishamian, M. S., Mirtaheri, S. A. \& Liu, J.-M. Ultracompact plasmonic loop-stub notch filter and sensor. Sens. Actuators B Chem. 194, 311-318 (2014).

46. Chen, W.-C. et al. Role of surface electromagnetic waves in metamaterial absorbers. Opt Express 24, 6783-6792. https://doi.org/ 10.1364/OE.24.006783 (2016).

47. Chau, Y.-F., Yeh, H.-H. \& Tsai, D. P. Surface plasmon effects excitation from three-pair arrays of silver-shell nanocylinders. Phys. Plasmas 16, 022303. https://doi.org/10.1063/1.3068469 (2009).

48. Chau, Y.-F.C. et al. Plasmonic effects in composite metal nanostructures for sensing applications. J. Nanoparticle Res. $20,190$. https://doi.org/10.1007/s11051-018-4293-4 (2018).

49. Chau, Y.-F., Liu, C.-Y., Yeh, H.-H. \& Tsai, D. P. A comparative study of high birefringence and low confinement loss photonic crystal fiber employing elliptical air holes in fiber cladding with tetragonal lattice. Progress Electromagnet. Res. 22, 39-52 (2010).

50. Chau, Y. F., Yeh, H. H. \& Tsai, D. P. Significantly enhanced birefringence of photonic crystal fiber using rotational binary unit cell in fiber cladding. J. Japan. J. Appl. Phys. 46, L1048-L1051 (2007).

51. West, P. R. et al. Searching for better plasmonic materials. Laser Photon. Rev. 4, 795-808. https://doi.org/10.1002/lpor.200900055 (2010).

52. Johnson, P. B. \& Christy, R. W. Optical constants of the noble metals. Phys. Rev. B 6, 4370-4379. https://doi.org/10.1103/PhysR evB.6.4370 (1972).

53. Chen, C., Oh, S.-H. \& Li, M. Coupled-mode theory for plasmonic resonators integrated with silicon waveguides towards midinfrared spectroscopic sensing. Opt. Express 28, 2020-2036. https://doi.org/10.1364/OE.28.002020 (2020).

54. Lu, H., Liu, X., Mao, D. \& Wang, G. Plasmonic nanosensor based on Fano resonance in waveguide-coupled resonators. Opt. Lett. 37, 3780-3782. https://doi.org/10.1364/OL.37.003780 (2012).

55. Li, M., Wang, J., Zhuang, L. \& Chou, S. Y. Fabrication of circular optical structures with a $20 \mathrm{~nm}$ minimum feature size using nanoimprint lithography. Appl. Phys. Lett. 76, 673-675 (2000).

56. Kamada, S., Okamoto, T., El-Zohary, S. E. \& Haraguchi, M. Design optimization and fabrication of Mach- Zehnder interferometer based on MIM plasmonic waveguides. Opt. Express 24, 16224-16231. https://doi.org/10.1364/OE.24.016224 (2016).

57. Kuttge, M., García de Abajo, F. J. \& Polman, A. Ultrasmall mode volume plasmonic nanodisk resonators. Nano Lett. 10, 1537-1541. https://doi.org/10.1021/nl902546r (2010).

58. Walther, C., Scalari, G., Amanti, M. I., Beck, M. \& Faist, J. Microcavity laser oscillating in a circuit-based resonator. Science 327, 1495-1497. https://doi.org/10.1126/science.1183167 (2010).

59. Yu, J., Zhu, J., Ye, S. \& Wang, X. Ultra-wide sensing range plasmonic refractive index sensor based on a two-dimensional circularhole grating engraved on a gold film. Results Phys. 26, 104396. https://doi.org/10.1016/j.rinp.2021.104396 (2021).

60. Chau, Y. F., Yeh, H. H. \& Tsai, D. P. A new type of optical antenna: Plasmonics nanoshell bowtie antenna with dielectric hole. J. Electromagnet. Waves Appl. 24, 1621-1632. https://doi.org/10.1163/156939310792149588 (2010).

61. Chau, Y.-F. \& Jiang, Z.-H. Plasmonics effects of nanometal embedded in a dielectric substrate. Plasmonics 6, 581-589. https://doi. org/10.1007/s11468-011-9238-z (2011).

62. Chou Chau, Y.-F. et al. Tunable optical performances on a periodic array of plasmonic bowtie nanoantennas with hollow cavities. Nanosc. Res. Lett. 11, 411. https://doi.org/10.1186/s11671-016-1636-x (2016).

63. Ballardin, C., Pereira-Stabile, C. L. \& Stabile, G. A. V. Use of a generic violet light in the surgical management of medication-related osteonecrosis of the jaws: a technical note. Oral. Maxillofac. Surg. 22, 477-481. https://doi.org/10.1007/s10006-018-0718-6 (2018).

64. Lago, A. D. N., Ferreira, W. D. R. \& Furtado, G. S. Dental bleaching with the use of violet light only: Reality or future?. Photodiagnosis Photodyn. Ther. 17, 124-126 (2017).

65. Murdoch, L. E., McKenzie, K., Maclean, M., Macgregor, S. J. \& Anderson, J. G. Lethal effects of high-intensity violet 405-nm light on Saccharomyces cerevisiae, Candida albicans, and on dormant and germinating spores of Aspergillus niger. Fungal Biol. 117, 519-527. https://doi.org/10.1016/j.funbio.2013.05.004 (2013).

66. Mimasaka, S., Oshima, T. \& Ohtani, M. Visualization of old bruises in children: Use of violet light to record long-term bruises. Forensic Sci. Int. 282, 74-78. https://doi.org/10.1016/j.forsciint.2017.11.015 (2018).

67. Wei, W., Zhang, X. \& Ren, X. Plasmonic circular resonators for refractive index sensors and filters. Nanosc. Res. Lett. 10, 1-6 (2015).

68. Butt, A., Khonina, S. \& Kazanskiy, N. Metal-insulator-metal nano square ring resonator for gas sensing applications. Waves Random Complex Media 31, 146-156. https://doi.org/10.1080/17455030.2019.1568609 (2019).

69. Kim, S. et al. Color-sensitive and spectrometer-free plasmonic sensor for biosensing applications. Biosensors Bioelectron. https:// doi.org/10.1016/j.bios.2018.11.048 (2018).

70. Song, H. et al. Comparison of fabrication methods based on nanoimprinting lithography for plasmonic color filter fabrication. Plasmonics 15, 941-948. https://doi.org/10.1007/s11468-019-01109-2 (2020).

71. Han, Z. \& Bozhevolnyi, S. I. Plasmon-induced transparency with detuned ultracompact Fabry-Perot resonators in integrated plasmonic devices. Opt. Express 19, 3251-3257. https://doi.org/10.1364/OE.19.003251 (2011).

72. Nozaki, K. et al. Ultralow-energy and high-contrast all-optical switch involving Fano resonance based on coupled photonic crystal nanocavities. Opt. Express 21, 11877-11888. https://doi.org/10.1364/OE.21.011877 (2013).

73. Huaiqing, L. et al. Ultrafast and low-power all-optical switch based on asymmetry electromagnetically induced transparency in MIM waveguide containing Kerr material. Opt. Commun. 353, 189-194. https://doi.org/10.1016/j.optcom.2015.05.018 (2015).

74. Negahdari, R., Rafiee, E. \& Emami, F. Realization of all-optical plasmonic MIM split square ring resonator switch. Opt. Quant. Electron. 51, 235. https://doi.org/10.1007/s11082-019-1924-7 (2019).

75. Bazgir, M., Jalalpour, M., Zarrabi, F. B. \& Arezoomand, A. S. Design of an optical switch and sensor based on a MIM Coupled Waveguide Using a DNA composite. J. Electron. Mater. 49, 2173-2178 (2020).

76. Liu, H. et al. Ultrafast and low-power all-optical switch based on asymmetry electromagnetically induced transparency in MIM waveguide containing Kerr material. Opt. Commun. 353, 189-194. https://doi.org/10.1016/j.optcom.2015.05.018 (2015).

77. Abbasi, F., Ghorashi, S. M. B., Karimzadeh, E. \& Zabolian, H. Investigating the effect of Ag and Au nanostructures with spherical and rod shapes on the emission wavelength of OLED. Plasmonics https://doi.org/10.1007/s11468-021-01441-6 (2021).

78. Chen, F. \& Yao, D. Tunable multiple all-optical switch based on multi-nanoresonator-coupled waveguide systems containing Kerr material. Opt. Commun. 312, 143-147. https://doi.org/10.1016/j.optcom.2013.09.011 (2014). 
79. Patskovsky, S., Kabashin, A. V., Meunier, M. \& Luong, J. H. T. Near-infrared surface plasmon resonance sensing on a silicon platform. Sens. Actuators B Chem. 97, 409-414. https://doi.org/10.1016/j.snb.2003.09.023 (2004).

80. She, S. et al. Fano-resonance-based refractive index sensor with ultra-high sensitivity. Results Phys. 25, 104327. https://doi.org/10. 1016/j.rinp.2021.104327 (2021).

81. Rakhshani, M. R. \& Mansouri-Birjandi, M. A. High sensitivity plasmonic refractive index sensing and its application for human blood group identification. Sens. Actuators B Chem. 249, 168-176. https://doi.org/10.1016/j.snb.2017.04.064 (2017).

82. Jha, R. \& Sharma, A. Design of a silicon-based plasmonic biosensor chip for human blood-group identification. Sens. Actuators B-Chem. 145, 200-204. https://doi.org/10.1016/j.snb.2009.11.060 (2010).

83. Li, H., Lin, L. \& Xie, S. Laser-Tissue Interaction XI: Photochemical, Photothermal, and Photomechanical 517-521 (International Society for Optics and Photonics, 2000).

84. Gienger, J., Smuda, K., Müller, R., Bär, M. \& Neukammer, J. Refractive index of human red blood cells between $290 \mathrm{~nm}$ and 1100 nm determined by optical extinction measurements. Sci. Rep. 9, 4623. https://doi.org/10.1038/s41598-019-38767-5 (2019).

85. Liu, S. et al. Measurement of the refractive index of whole blood and its components for a continuous spectral region. J. Biomed. Opt. 24, 1-5. https://doi.org/10.1117/1.JBO.24.3.035003 (2019).

86. Zhernovaya, O., Sydoruk, O., Tuchin, V. \& Douplik, A. The refractive index of human hemoglobin in the visible range. Phys. Med. Biol. 56, 4013-4021. https://doi.org/10.1088/0031-9155/56/13/017 (2011).

87. Friebel, M. \& Meinke, M. Model function to calculate the refractive index of native hemoglobin in the wavelength range of 250-1100 nm dependent on concentration. Appl. Opt. 45, 2838-2842. https://doi.org/10.1364/AO.45.002838 (2006).

88. Sharma, A. K. Plasmonic biosensor for detection of hemoglobin concentration in human blood: Design considerations. J. Appl. Phys. 114, 044701. https://doi.org/10.1063/1.4816272 (2013).

89. Li, H., Lin, L. \& Xie, S. Refractive Index of Human Whole Blood with Different Types in the Visible and Near-Infrared Ranges Vol. 3914 (SPIE, 2000).

\section{Acknowledgements}

This research was supported by the University Research Grant of Universiti Brunei Darussalam (Grant no. UBD/ RSCH/1.9/FICBF(b)/2021/009) and the Ministry of Science and Technology of Taiwan (MOST 110-2112-M019-004 and MOST 109-2112-M-415-003).

\section{Author contributions}

Y.F.C.C. investigation, resources, conceptualization and writing. C.T.C.C. investigation, simulation, and data analysis, H.J.H. and S.H.C. methodology, writing-review and editing, T.S.K. and H.P.C. conceptualization, methodology, writing-review and editing.

\section{Competing interests}

The authors declare no competing interests.

\section{Additional information}

Correspondence and requests for materials should be addressed to Y.-F.C.C., T.S.K. or H.-P.C.

Reprints and permissions information is available at www.nature.com/reprints.

Publisher's note Springer Nature remains neutral with regard to jurisdictional claims in published maps and institutional affiliations.

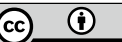

Open Access This article is licensed under a Creative Commons Attribution 4.0 International License, which permits use, sharing, adaptation, distribution and reproduction in any medium or format, as long as you give appropriate credit to the original author(s) and the source, provide a link to the Creative Commons licence, and indicate if changes were made. The images or other third party material in this article are included in the article's Creative Commons licence, unless indicated otherwise in a credit line to the material. If material is not included in the article's Creative Commons licence and your intended use is not permitted by statutory regulation or exceeds the permitted use, you will need to obtain permission directly from the copyright holder. To view a copy of this licence, visit http://creativecommons.org/licenses/by/4.0/.

(C) The Author(s) 2021 